\title{
RENAL TUBULAR IMPAIRMENT IN CHILDREN WITH IDIOPATHIC HYPERCALCIURIA
}

\author{
Sylva Skálová1, Štěpán Kutílek \\ Charles University in Prague, Faculty of Medicine and University Hospital in Hradec Králové, Czech Republic; \\ Department of Pediatrics ${ }^{1}$, Center for Clinical and Basic Research, Pardubice, Czech Republic ${ }^{2}$
}

Summary: Idiopathic hypercalciuria (IH) is defined as hypercalciuria that persists after correction of dietary inbalances and has no detectable cause. The excretion of urinary N-acetyl-beta-D-glucosaminidase (U-NAG), a marker of proximal tubular damage, has been previously reported as either increased or normal in children with IH. We evaluated U-NAG in 20 children ( 13 boys and 7 girls, mean age 10.3 years $\pm 5.7 \mathrm{SD}$ ) with $\mathrm{IH}$ (urinary calcium excretion above $0.1 \mathrm{mmol} / \mathrm{kg} / 24$ hours, with no detectable cause) and with otherwise normal renal function tests. Ultrasound examination revealed urolithiasis $(n=4)$ and nephrocalcinosis $(n=1)$. The U-NAG values were evaluated in the spot urine collected from the second morning void and calculated as the urinary NAG/creatinine ratio (U-NAG/Cr) and expressed in nkat $/ \mathrm{mmol}$. The 24-hour urinary calcium excretion (U-Ca/24h) was assessed in a urinary sample from 24-hour collected urine and calculated in $\mathrm{mmol} / \mathrm{kg}$. The obtained results of $\mathrm{U}-\mathrm{Ca} / 24 \mathrm{~h}$ and $\mathrm{U}-\mathrm{NAG} / \mathrm{Cr}$ were expressed as Z-scores. When compared to the reference data, the U-Ca/24h and U-NAG/Cr were significantly higher ( $p=0.0004$ and $\mathrm{p}=0.006$, respectively). There was no correlation between the U-NAG/Cr and U-Ca/24h $(r=0.18, p=0.20)$. The U-NAG/Cr values were significantly higher in the 5 patients with urolithiasis/nephrocalcinosis, whether compared to the rest of the group ( $p=0.02$ ), or to the reference data $(\mathrm{p}=0.01)$. The U-NAG/Cr activity was higher in 15 children without urolithiasis/nephrocalcinosis when compared to reference data $(\mathrm{p}<0.01)$. There was no difference in $\mathrm{U}-\mathrm{Ca} / 24 \mathrm{~h}$ between the children with and without urolithiasis/nephrocalcinosis $(\mathrm{p}=0.58)$. These findings suggest that tubular impairment, as reflected by U-NAG/Cr, might occur in children with IH, especially in patients with urolithiasis/nephrocalcinosis. There doesn't seem to be a direct relationship between the U-NAG/Cr activity and the degree of calcium leakage.

Key words: Urinary NAG; Idiopathic hypercalciuria

\section{Introduction}

Idiopathic hypercalciuria (IH) is defined as hypercalciuria in the presence of normocalcemia, that persists after correction of dietary inbalances with no detectable cause, and whose clinical manifestation varies with age (3). Renal tubular dysfunction seems as less likely the primary cause of IH $(3,6,9)$. However, renal tubular impairment can be encountered in patients with urolithiasis or nephrocalcinosis, as cell-crystal interactions may lead to tubular damage and/or dysfunction $(1,6,10)$. In children with IH there is an age-dependent risk of formation of microcalculi or stones, and development of osteoporosis $(4,5,8)$. The excretion of urinary N-acetyl-beta-D-glucosaminidase (U-NAG), a marker of proximal tubular damage, has been reported as either increased (8-10), or normal (1), in children with IH. Recently, U-NAG was reported as significantly higher in children with urolithiasis and nephrocalcinosis, but not in children with isolated $\mathrm{IH}$ alone, and did not correlate with the urinary excretion of oxalate or calcium (6). Therefore, we look- ed for relationship between calciuria and U-NAG in children with $\mathrm{IH}$.

\section{Patients, Materials, Methods}

\section{Patients}

We enrolled 20 children ( 13 boys and 7 girls, mean age 10.3 years $\pm 5.7 \mathrm{SD}$ ) with IH (urinary calcium excretion above $0.1 \mathrm{mmol} / \mathrm{kg} / 24$ hours, with no detectable cause) and with otherwise normal renal function tests and normal values of serum calcium, phosphate, alkaline phosphatase and parathyroid hormone. These children were referred because of hematuria and abdominal pain. Ultrasound examination revealed urolithiasis $(n=4)$ and nephrocalcinosis $(n=1)$.

\section{Materials and Methods}

For the evaluation of U-NAG and calciuria, the urine was collected on the same day. The 24-hour urinary calcium excretion ( $\mathrm{U}-\mathrm{Ca} / 24 \mathrm{~h}$ ) was assessed in a urinary sample from 24-hour collected urine by means of photometry and calcu- 


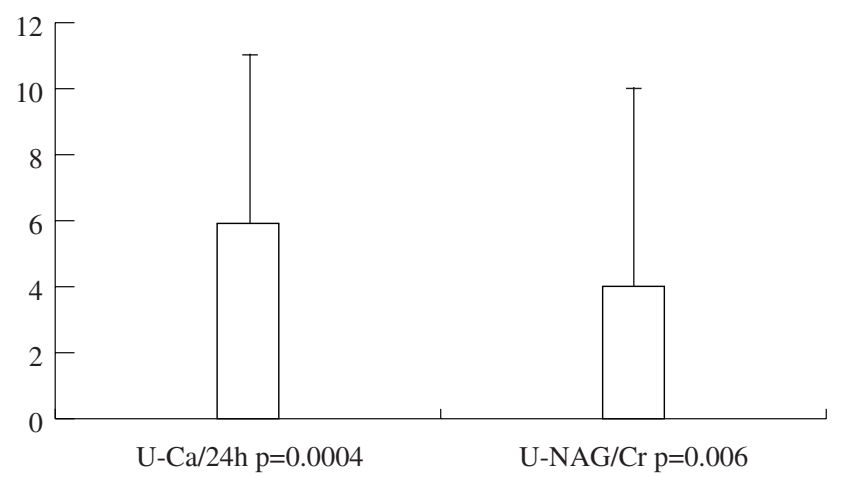

Fig. 1: 24-hour urinary calcium excretion (U-Ca/24h) and urinary N-acetyl-beta-D-glucosaminidase activity/creatinine (U-NAG/Cr) in 20 children with idiopathic hypercalciuria (expressed as Z-scores $\pm \mathrm{SD}$, compared to reference data).

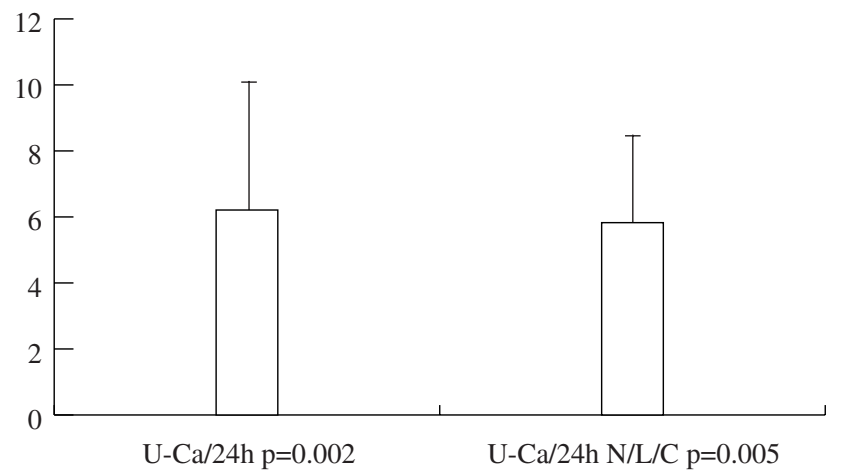

Fig. 2: The U-Ca/24h values in children without urolithiasis/nephrocalcinosis $(n=15)$ and with urolithiasis/nephrocalcinosis $(\mathrm{N} / \mathrm{L} / \mathrm{C})(\mathrm{n}=5)$ (expressed as Z-scores $\pm \mathrm{SD}$, compared to reference data). There was no diference in $\mathrm{U}-\mathrm{Ca} / 24 \mathrm{~h}$ between the children with and without urolithiasis/nephrocalcinosis $(p=0.58)$.

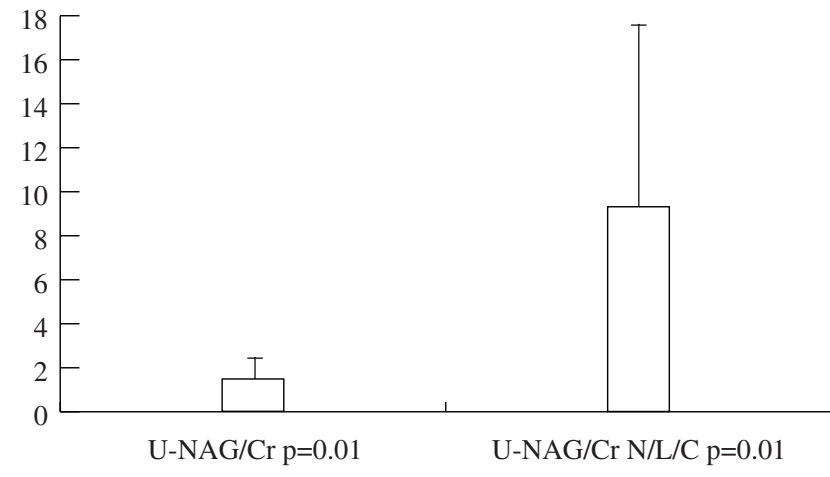

Fig. 3: The U-NAG/Cr values in patients without urolithiasis/nephrocalcinosis $(\mathrm{n}=15)$ and with urolithiasis/nephrocalcinosis $(\mathrm{N} / \mathrm{L} / \mathrm{C})(\mathrm{n}=5)($ expressed as Z-scores $\pm \mathrm{SD}$, compared to reference data). The U-NAG/Cr values were significantly higher in the 5 patients with urolithiasis/nephrocalcinosis when compared to patients without urolithiasis/nephrocalcinosis $(p=0.02)$. lated in $\mathrm{mmol} / \mathrm{kg}$. The catalytic activity of NAG was measured by fluorimetric assay in spot urine collected from the second morning void. The influence of endogenous enzyme inhibitors was eliminated by diluting the urine specimens 20-fold. The urinary creatinine concentration was estimated by Jaffe's kinetic method on Modular Analyser (Roche Diagnostics GmbH, Sandhofer Strasse 116, Mannheim, Germany). The U-NAG values were calculated as the urinary NAG/creatinine ratio (U-NAG/Cr) and expressed in nkat $/ \mathrm{mmol}$. To eliminate the influence of age, the obtained results of $\mathrm{U}-\mathrm{Ca} / 24 \mathrm{~h}$ and $\mathrm{U}-\mathrm{NAG} / \mathrm{Cr}$ were calculated as Z-scores by the equation SDS = (actual individual value - mean value for age) / standard deviation (SD) for age. The reference values for U-NAG/Cr and U-Ca/24h were represented by the previously published data of healthy European paediatric populations $(2,7)$.

\section{Statistical Evaluation}

The statistical evaluation was performed by t-test, ANOVA and linear regression analysis. For all results, $p<0.05$ was required for statistical significance.

\section{Results}

\section{$U-C a / 24 h$}

When compared to the reference data, the U-Ca/24h was significantly higher (Fig. 1). The U-Ca/24h was significantly higher in comparison to the reference data either in patients without urolithiasis/nephrocalcinosis $(n=15)$ or with urolithiasis/nephrocalcinosis $(n=5)$ (Fig. 2). However, there was no difference in $\mathrm{U}-\mathrm{Ca} / 24 \mathrm{~h}$ between the children without urolithiasis/nephrocalcinosis and with urolithiasis/ nephrocalcinosis (Fig. 2).

\section{$U-N A G / C r$}

In comparison to the reference data, the U-NAG/Cr was significantly higher (Fig. 1). In 7 patients (35\%), the U-NAG/Cr values exceeded the 95th percentile of the agerelated reference range. Furthermore, regarding children with urolithiasis/nephrocalcinosis $(n=5), 3$ patients had the U-NAG/Cr values above the 95th percentile. The U-NAG/ $\mathrm{Cr}$ values were significantly higher in the 5 patients with urolithiasis/nephrocalcinosis, whether compared to the rest of the group or to the reference data. The U-NAG/Cr activity was still higher in 15 children without urolithiasis/ nephrocalcinosis when compared to reference data (Fig. 3).

Relationship between $\mathrm{U}$-Ca/24h and $\mathrm{U}-\mathrm{NAG} / \mathrm{Cr}$

There was no correlation between the U-NAG/Cr and U-Ca/24h $(r=0.18, p=0.20)$.

\section{Discussion}

Our findings suggest that tubular impairment, as reflected by U-NAG/Cr, might occur in children with $\mathrm{IH}$, especially in patients with urolithiasis/nephrocalcinosis. The absence of correlation between $\mathrm{U}-\mathrm{NAG} / \mathrm{Cr}$ and $\mathrm{U}-\mathrm{Ca} /$ 
$24 \mathrm{~h}$ also suggests that renal tubular impairment really seems to be less likely the primary cause of $\mathrm{IH}(3,6,8,9)$. However, in contrast to the findings of Sikora et al (6), we found increased U-NAG/Cr even in patients with $\mathrm{IH}$ and without urolithiasis/nephrocalcinosis. Therefore, increased urinary concentration of calcium might lead to damage of tubular cells, even in the absence of lithiasis. Furthermore, the findings of higher U-NAG/Cr in patients with urolithiasis/nephrocalcinosis in comparison to the values in children with IH but without urolithiasis/nephrocalcinosis further support the hypothesis that cell-crystal interactions lead to tubular impairment. In conclusion, children with IH have some degree of secondary renal tubular impairment. The tubular impairment is most probably aggravated by the increased urinary concentration of calcium, and, in particular, by the cell-crystal interactions. However, there doesn't seem to be a direct relationship between this tubular impairment and the degree of calcium leakage.

\section{Acknowledgements}

We are most grateful to prof. V. Palička and his team from the Institute of Clinical Biochemistry and Diagnosis at the Faculty of Medicine in Hradec Králové for performing the U-Ca/24h and U-NAG/Cr measurements.

\section{References}

1. Kavukcu S, Aydin A, Turkmen M, Akhunlar H, Fadiloglu M, Tavli V. Investigation of relationship between idiopathic hypercalciuria and urinary enzyme activities. J Assoc Physicians India 1998;46:784-5.
2. Manz F, Keprt R, Larsen B, Merkem A. Urinary calcium excretion in healthy children and adolescents. Pediatr Nephrol 1999;13:894-9.

3. Penido MG, Diniz JS, Moreira ML, Tupinamba AL, Franca A, Andrade BH, et al. Idiopathic hypercalciuria: presentation of 471 cases. J Pediatr (Rio J). 2001;77:101-4

4. Penido MG, Lima EM, Marino VS, Tupinamba AL, Franca A, Souto MF. Bone alterations in children with idiopathic hypercalciuria at the time of diagnosis. Pediatr Nephrol 2003; 18:133-9.

5. Polito C, La Manna A, Cioce F, Villani J, Nappi B, Di Toro R. Clinical presentation and natural course of idiopathic hypercalciuria in children. Pediatr Nephrol 2000;15:211-4

6. Sikora P, Glatz S, Beck BB, Stapenhorst L, Zajaczkowska M, Hesse A, et al. Urinary NAG in children with urolithiasis, nephrocalcinosis, or risk of urolithiasis. Pediatr Nephrol 2003;18:996-9.

7. Skálová S, Chládek J. Urinary N-acetyl-beta-D-glucosaminidase activity in healthy children. Nephrology 2004;9:19-21.

8. Skálová S, Palička V, Kutílek Š. Bone mineral density and urinary N-acetyl-betaD-glucosaminidase activity in paediatric patients with idiopathic hypercalciuria. Nephrology 2005;10:99-102.

9. Stapleton FB, Chesney RW, Behrmann AT, Miller LA. Increased urinary excretion of renal N-acetyl-beta-glucosaminidase in hypercalciuria. Am J Dis Child 1985;139:950-2.

10. Tekin N, Kural N, Torun M. Renal function in children with hypercalciuria. Turk J Pediatr 1997;39:335-9.

Submitted August 2005.

Accepted April 2006.

Sylva Skálová, M.D.,

University Hospital,

Department of Pediatrics, Sokolská 581,

50005 Hradec Králové, Czech Republic.

e-mail: skalova.s@seznam.cz 